\title{
Correction to: Systematic review of pre-clinical chronic myeloid leukaemia
}

\author{
Jew Win Kuan ${ }^{1}\left[\right.$. Anselm Ting $\mathrm{Su}^{2} \cdot$ Chooi Fun Leong $^{3} \cdot$ Motomi Osato $^{4} \cdot$ Goro Sashida ${ }^{4}$
}

Published online: 7 November 2018

(c) The Japanese Society of Hematology 2018

\section{Correction to: \\ International Journal of Hematology (2018) 108:465-484 \\ https://doi.org/10.1007/s12185-018-2528-x}

The author would like to correct the error in the publication of the original article. The corrected detail is given below for your reading:

The first sentence in the last paragraph on page 481 in "Discussion" section under the subheading "Overall completeness and applicability of evidence" should read as "Clonal haematopoiesis of indeterminate potential (CHIP) was first named in 2015 [27]."

The original article can be found online at https://doi.org/10.1007/ s12185-018-2528-x.

Jew Win Kuan

kuanjewwin@gmail.com

1 Department of Medicine, Faculty of Medicine and Health Sciences, Universiti Malaysia Sarawak, 94300 Kota Samarahan, Sarawak, Malaysia

2 Department of Community Medicine and Public Health, Faculty of Medicine and Health Sciences, Universiti Malaysia Sarawak, Kota Samarahan, Sarawak, Malaysia

3 Department of Pathology, Universiti Kebangsaan Malaysia Medical Center, Kuala Lumpur, Malaysia

4 International Research Center for Medical Sciences, Kumamoto University, Kumamoto, Japan 